Option Pricing in a Multi-Asset, Complete Market Economy

Author(s): Ren-Raw Chen, San-Lin Chung, Tyler T. Yang

Source: The Journal of Financial and Quantitative Analysis, Vol. 37, No. 4 (Dec., 2002), pp. 649 $-666$

Published by: University of Washington School of Business Administration

Stable URL: http://www.jstor.org/stable/3595015

Accessed: 10/09/2008 21:42

Your use of the JSTOR archive indicates your acceptance of JSTOR's Terms and Conditions of Use, available at http://www.jstor.org/page/info/about/policies/terms.jsp. JSTOR's Terms and Conditions of Use provides, in part, that unless you have obtained prior permission, you may not download an entire issue of a journal or multiple copies of articles, and you may use content in the JSTOR archive only for your personal, non-commercial use.

Please contact the publisher regarding any further use of this work. Publisher contact information may be obtained at http://www.jstor.org/action/showPublisher?publisherCode=uwash.

Each copy of any part of a JSTOR transmission must contain the same copyright notice that appears on the screen or printed page of such transmission.

JSTOR is a not-for-profit organization founded in 1995 to build trusted digital archives for scholarship. We work with the scholarly community to preserve their work and the materials they rely upon, and to build a common research platform that promotes the discovery and use of these resources. For more information about JSTOR, please contact support@jstor.org. 


\title{
Option Pricing in a Multi-Asset, Complete Market Economy
}

\author{
Ren-Raw Chen, San-Lin Chung, and Tyler T. Yang*
}

\section{Abstract}

This paper extends the seminal Cox-Ross-Rubinstein ((1979), CRR hereafter) binomial model to multiple assets. It differs from previous models in that it is derived under the complete market environment specified by Duffie and Huang (1985) and He (1990).

The complete market assumption requires the number of states to grow linearly with the number of assets. However, the number of correlations grows at a faster rate, causing the CRR model to be indirectly extendable. We solve such a problem by recognizing that the fast growing correlation number is matched by the number of the angles of the edges of a hypercube spanned by the risky assets. As a result, we derive a solution that allows the number of equations to equal the number of risky assets and the riskless bond. The resulting tree structure hence provides the same intuition of pricing and hedging contingent claims as that provided by the CRR model.

Finally, the proposed model is not only as easy to implement as the one-dimensional CRR model but also it is more memory efficient than the existing multi-factor lattice models.

\section{Introduction}

The contribution of the Cox-Rubinstein-Ross (CRR hereafter) binomial model (1979) is two-fold. ${ }^{1}$ It provides a method to price American options in the Black-Scholes world and it demonstrates how arbitrage can function in a dynamically complete economy. Within this economy, the market is complete and hence the option is a redundant asset and can be duplicated by the stock and the riskfree bond. A formal description of such an economy is provided by Duffie and Huang (1985). He (1990) demonstrated a multi-asset version of the Duffie-Huang model. To obtain market completeness in a $k$-asset world, the number of states at

\footnotetext{
*Chen, rchen@rci.rutgers.edu, Rutgers Business School, Rutgers University, 94 Rockafeller Road, Piscataway, NJ 08854; Chung, chungs@mgt.ncu.edu.tw, Department of Finance, National Central University, Taiwan, R.O.C.; and Yang, tyler.yang@ifegroup.com, IFE Group, 51 Monroe Street, Plaza E6, Rockville, MD 20850. We thank Peter Carr and the participants of the seminars held by Rutgers University and Hong Kong University of Sciences and Technologies for their valuable suggestions. We are especially indebted to Frank Milne and Avi Bick (the referees) for their very helpful suggestions that improved the paper's content and the exposition. Chen thanks the Faculty of Management of Rutgers University for financial support.

${ }^{1}$ An identical model also appeared in Rendleman and Bartter (1979). William Sharpe (1976) was the first to provide the arbitrage-free portfolio.
} 
each period can only be $k+1$. He also proved that such a model can converge to a multivariate version of the Black-Scholes model.

Hull and White (1990) showed that the binomial model is a special case of the explicit finite difference method for solving the partial differential equation. The usual explicit finite difference method, or the trinomial model, assumes three states of economy for every period. Unfortunately, due to the extra state, the trinomial tree cannot support the arbitrage-free argument as the CRR binomial model does.

There have been several extensions of the CRR model to incorporate multiple underlying assets. ${ }^{2}$ Boyle (1988) first developed an algorithm for American options on two correlated assets. ${ }^{3}$ Given that four branches provide the most straightforward way to use the binomial model for two uncorrelated assets, Boyle added a fifth branch to handle the correlation.

Boyle, Evnine, and Gibbs (1989) extended the Boyle model to $k$ factors. They use $2^{k}$ branches for each step under $k$ factors. The correlations are matched by properly adjusting the probability assigned to each branch. Kamrad and Ritchken (1991) improved the efficiency of the Boyle-Evnine-Gibbs model by incorporating more branches. Ho, Stapleton, and Subrahmanyam (1995) carried the Boyle-Evnine-Gibbs model one step further to include a time-varying variancecovariance structure. However, with this more complicated structure, they can only value Bermudan options, options that can be exercised only at selected points in time. Recently, MaCarthy and Webber (1999) argued that in high dimensional lattices, nodes tend to be placed in a very inefficient way. Using a two-asset example, they argued that efficiency can be significantly improved if a six-branch hexagon lattice is used.

Our model differs from the existing models in that it is more than a numerical algorithm for pricing American style options under multiple risky assets. It is a model that is arbitrage free in every period - the result only achievable under complete markets. Furthermore, only such a model can avoid complex optimization in its implementation. The model we propose in this paper has wider applications in asset pricing and can be extended to include orthonormal Poisson processes (see Milne and Turnbull (1997)). ${ }^{4}$ Finally, as a byproduct, the proposed model is more memory efficient than the previous models.

The difficulty in handling correlations in higher dimensions under complete markets is that the number of correlations grows at an accelerating speed, i.e., $C_{2}^{k}=(k(k-1)) / 2$ for $k$ risky assets while the number of assets grows linearly. In a two-asset model, two means, one correlation, and two variances provide five equations that are unable to uniquely identify six unknown states (three nodes with two coordinates). This problem becomes more serious in higher dimensions.

\footnotetext{
${ }^{2}$ Other related (but not directly) studies include Amin (1991) who demonstrated several methods to approximate the multivariate lognormal process, Barraquand and Martineau (1995) who used Monte Carlo to price American options with multiple assets, Boyle and Tse (1990) who priced European options with multiple assets, and Madan, Milne, and Shefrin (1989) who used a multi-branch lattice to price American options with a single asset.

${ }^{3}$ There are lattice models that deal with multiple factors, e.g., Hull and White (1990) and Chen and Scott (1992), but those models assume uncorrelated factors. For numerical methods used in pricing derivatives in general, see also Clewlow and Strickland (1999) and Tavella and Randall (2000).

${ }^{4}$ We thank Frank Milne for pointing this out to us.
} 
Since there are always fewer equations, $k$ means, and $k$ variances plus $(k(k-$ 1)) $/ 2$ correlations than number of states, $k(k+1)$, the nodes cannot be identified uniquely.

In this paper, we derive a solution that allows the number of equations to be equal to the number of risky assets and the riskless bond. We leverage upon the geometrical structure of the ellipse to demonstrate the model in two dimensions and extend the structure to $k$ dimensions. Our model therefore can be viewed as a direct extension of the CRR model to a multi-asset economy where the complete market property is preserved. Despite using fewer nodes, the model is as accurate as the existing lattice models.

The paper is organized as follows. Section II derives the structure of a twoasset economy that establishes market completeness and demonstrates the implementation of the model in three and higher dimensions in the Appendix. Numerical examples presented in Section III show the convergence and the results are compared with those of Margrabe (1978), Boyle (1988), and Boyle, Evnine, and Gibbs (1989). The last section summarizes the contribution of the paper.

\section{The Model}

We begin our model by briefly reviewing the concept of a complete market. In a complete market economy, options are redundant assets and can be replicated by primary securities, namely stocks and a risk-free bond. Hence, options can be priced by arbitrage in which risk premiums are not relevant. The necessary condition for the arbitrage-free argument to hold is that at any point in time, the option payoff can be perfectly replicated by a synthetic call composed of $k$ stocks and $\$ 1$ cash, as follows,

$$
\left[\begin{array}{ccccc}
S_{1,1} & S_{2,1} & \cdots & S_{k, 1} & 1 \\
S_{1,2} & S_{2,2} & \cdots & S_{k, 2} & 1 \\
\vdots & \vdots & \ddots & \vdots & \vdots \\
S_{1, m-1} & S_{2, m-1} & \cdots & S_{k, m-1} & 1 \\
S_{1, m} & S_{2, m} & \cdots & S_{k, m} & 1
\end{array}\right] \times\left[\begin{array}{c}
\Delta_{1} \\
\Delta_{2} \\
\vdots \\
\Delta_{k} \\
\Delta_{B}
\end{array}\right]=\left[\begin{array}{c}
C_{1} \\
C_{2} \\
\vdots \\
C_{m}
\end{array}\right]
$$

where $S_{i j}$ represents the end-of-period price of stock $i$ at state $j, C_{j}$ represents the end-of-period call value at state $j$, and $\Delta_{i}$ represents the hedge ratio (or portfolio weight) for security $i$ where $i \in\{1, \ldots, k\}$ for stocks and $i=B$ for the risk-free bond. If the stock price matrix is full rank $(=m)$, then there is a unique solution for the hedge ratios and hence the option can be completely replicated by stocks and the bond.

Given the hedge ratios, the law of one price asserts that today's option price must be

$$
C_{0}=\left[S_{1} S_{2} \cdots S_{k} B\right] \times\left[\Delta_{1} \Delta_{2} \cdots \Delta_{k} \Delta_{B}\right]^{T},
$$

where $C_{0}$ is the current option price, $S_{i}$ is the current price of stock $i$, and $B$ is the current price of the risk-free zero coupon bond that pays $\$ 1$ at the end of the period. 
In the two-asset version of the Black-Scholes model, the dynamics for the two assets are

$$
\left\{\begin{array}{l}
d \ln S_{1}(t)=\left(r-\frac{\sigma_{1}^{2}}{2}\right) d t+\sigma_{1} d W_{1}(t) \\
d \ln S_{2}(t)=\left(r-\frac{\sigma_{2}^{2}}{2}\right) d t+\sigma_{2} d W_{2}(t),
\end{array}\right.
$$

where $r$ is the risk-free rate, $\sigma_{1}$ and $\sigma_{2}$ are the instantaneous standard deviations of $d S_{1}$ and $d S_{2}$, and $W_{1}$ and $W_{2}$ are two correlated risk-neutral Wiener processes where $d W_{1} d W_{2}=\rho d t$.

Our objective is to design the stock price matrix in equation (1) to match equation (3) in discrete time. To maintain a complete market, the tree structure of the above two-factor model can be graphically depicted.

\section{FIGURE 1}

The Two-Period Geometry of the Two-Factor Model

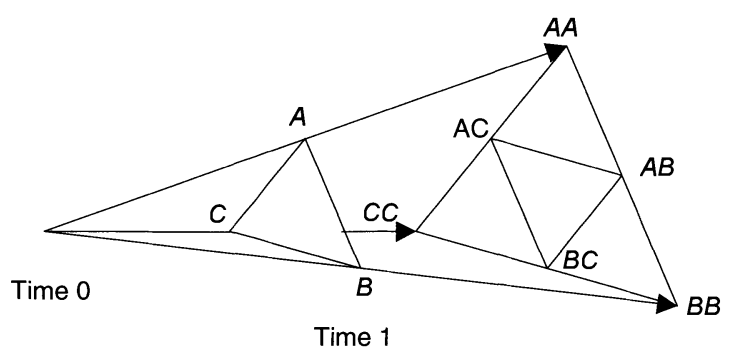

Time 2

Figure 1 is a three-dimensional diagram in which points $A, B$, and $C$ at time 1 are evolved from the point at time 0 and each is a vertical vector of a pair of values for two stocks. The three points at time 1 then evolve to six points at time 2 ( $A$ moves to $A A, A B$, and $A C ; B$ moves to $A B, B B$, and $B C$; and $C$ moves to $A C, B C$, and $C C$ for up, right, and left, respectively). The evolution continues until the maturity of the derivative asset. Taking the first period as an example, we can write the stock price evolution as

$$
\begin{aligned}
& {\left[\begin{array}{ll}
\ln S_{1,1}(1) & \ln S_{2,1}(1) \\
\ln S_{1,2}(1) & \ln S_{2,2}(1) \\
\ln S_{1,3}(1) & \ln S_{2,3}(1)
\end{array}\right]=\left[\begin{array}{l}
1 \\
1 \\
1
\end{array}\right]} \\
& \quad \times\left[\begin{array}{ll}
\ln S_{1}(0)+\left(r-\frac{\sigma_{1}^{2}}{2}\right) \Delta t & \ln S_{2}(0)+\left(r-\frac{\sigma_{2}^{2}}{2}\right) \Delta t
\end{array}\right]+\left[\begin{array}{c}
A \\
B \\
C
\end{array}\right],
\end{aligned}
$$


where $[A B C]^{T}$ is a $3 \times 2$ matrix (to be specified later) and $S_{i}(0)$, as previously defined, represents the price of stock $i$ at time $0, S_{i, j}(1)$ is the price of the $i$ th stock in the $j$ th state at time 1 . To keep the algebra more tractable, we assign equal probabilities (1/3) for the states. As described in He (1990), the complete market assumption allows for only three states, rather than four or five states in other numerical studies.

With two uncorrelated Brownian motions with equal variances, the three points, $A, B$, and $C$, in Figure 1 are best "equally" apart from each other. This can be achieved most easily by choosing three points, located $120^{\circ}$ from each other, on the circumference of a circle, as shown in Figure $2 .{ }^{5}$ It is not important, however, where the three points are. For example, $A^{\prime}, B^{\prime}$, and $C^{\prime}$ are another set of choices that can mimic the same two uncorrelated unit Brownian motions. ${ }^{6}$

\section{FIGURE 2}

\section{Three Points that Represent Two Orthogonal Brownian Motions}

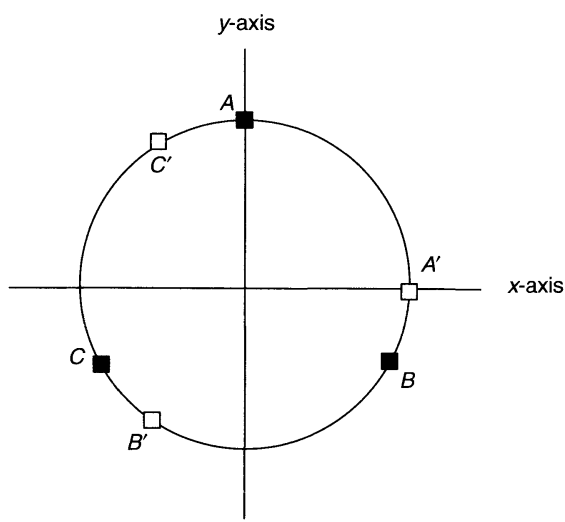

For two uncorrelated Brownian motions with different standard deviations, the circle in Figure 2 can be adjusted to be an upright ellipse,

$$
\left[\frac{x}{r_{x}}\right]^{2}+\left[\frac{y}{r_{y}}\right]^{2}=1,
$$

where $r_{x}$ and $r_{y}$ represent the two radiuses of the ellipse along the $x$-axis and $y$ axis, respectively. The following proposition states that the upright ellipse defined by equation (5) has different volatilities along the two axes and zero correlation.

\footnotetext{
${ }^{5}$ The coordinates are $(0,1),(\sqrt{3} / 2,-1 / 2)$, and $(-\sqrt{3} / 2,-1 / 2)$ for $A, B$, and $C$, respectively. Note that this choice of coordinates is also used by He (1990).

${ }^{6}$ The coordinates are $(1,0),(-1 / 2,-\sqrt{3} / 2)$, and $(-1 / 2, \sqrt{3} / 2)$ for $A^{\prime}, B^{\prime}$, and $C^{\prime}$, respectively.
} 
In what follows, we always assume that the states $1, \ldots, n$, have equal probabilities, and we identify $\left(x_{1}, \cdots, x_{n}\right)$ with the random variable $X$, which obtains value $x_{j}$ in state $j$ with probability $1 / n$. Thus, the mean and variance are

$$
E[X]=\frac{1}{n} \sum_{j=1}^{n} x_{j}, \quad V[X]=\frac{1}{n} \sum_{j=1}^{n}\left(x_{j}-E[X]\right)^{2},
$$

and the covariance is

$$
K[X, Y]=\frac{1}{n} \sum_{j=1}^{n}\left(x_{j}-E[X]\right)\left(y_{j}-E[Y]\right),
$$

where $Y$ is another random variable that obtains the values $\left(y_{1}, \cdots, y_{n}\right)$ in the same states $1, \ldots, n$.

Proposition 1 . Let $\left(x_{1}, y_{1}\right), \ldots,\left(x_{n}, y_{n}\right)$ be equally spread (angle-wise) $n$ points $(n \geq 3)$ on the ellipse defined by equation (5), where $r_{x}$ and $r_{y}$ are given positive numbers. Let $X$ and $Y$ be the random variables that obtain the values $\left(x_{1}, \cdots, x_{n}\right)$ and $\left(y_{1}, \cdots, y_{n}\right)$, respectively, with equal probabilities. Then, regardless of the location of the $n$ points, $X$ and $Y$ satisfy

$$
\begin{aligned}
E[X] & =E[Y]=0, \\
\sigma_{X}^{2} & =\operatorname{var}[X]=\frac{1}{2} r_{x}^{2}, \quad \sigma_{Y}^{2}=\operatorname{var}[Y]=\frac{1}{2} r_{y}^{2}, \\
K[X, Y] & =\operatorname{cov}[X, Y]=0 .
\end{aligned}
$$

Proof. For easy exposition, we first do change of variable to "normalize" random variables $X$ and $Y$ as

$$
\begin{aligned}
X^{*} & =\frac{X}{r_{x}} \\
Y^{*} & =\frac{Y}{r_{y}} .
\end{aligned}
$$

By doing this change of variable, we effectively transform the ellipse into a circle. The $n$ points along the circumference of a circle can be presented by polar coordinates as

$$
\left\{\begin{array}{l}
x_{j}^{*}=\cos \left(\theta+\frac{j 2 \pi}{n}\right) \\
y_{j}^{*}=\sin \left(\theta+\frac{j 2 \pi}{n}\right)
\end{array} \quad j=1, \ldots, n,\right.
$$

where $\theta$ is the angle of the starting point. The goal is to show that the correlation, the means, and the standard deviations are independent of $\theta$ and $n$.

The means of $X^{*}$ and $Y^{*}$ are

$$
\begin{aligned}
& E\left[X^{*}\right]=\frac{1}{n} \sum_{j=1}^{n} \cos \left(\theta+\frac{j 2 \pi}{n}\right)=0, \\
& E\left[Y^{*}\right]=\frac{1}{n} \sum_{j=1}^{n} \sin \left(\theta+\frac{j 2 \pi}{n}\right)=0
\end{aligned}
$$


which, after cancellation, ${ }^{7}$ are identically 0 and independent of $\theta$ and $n$. The variances are

$$
\begin{aligned}
& V\left[X^{*}\right]=\frac{1}{n} \sum_{j=1}^{n} x_{j}^{* 2}=\frac{1}{n} \sum_{j=1}^{n} \cos ^{2}\left(\theta+\frac{j 2 \pi}{n}\right)=\frac{1}{2}, \\
& V\left[Y^{*}\right]=\frac{1}{n} \sum_{j=1}^{n} y_{j}^{* 2}=\frac{1}{n} \sum_{j=1}^{n} \cos ^{2}\left(\theta+\frac{j 2 \pi}{n}\right)=\frac{1}{2},
\end{aligned}
$$

which imply that the variances of the original variables $X$ and $Y$ have variances that are equal to one-half multiplied by the squares of the radiuses. The last equality is obtained by using the following relationship: $\cos ^{2} \theta=1 / 2(\cos 2 \theta+1)$. As a result, $1 /(2 n) \Sigma_{j} \cos 2(\theta+j 2 \pi / n)=0$ and $1 /(2 n) \Sigma_{j} 1=1 / 2$ and the derivation is complete. ${ }^{8}$ Again, they are independent of $n$ and $\theta$. Finally, the covariance is

$$
\begin{aligned}
K\left[X^{*}, Y^{*}\right] & =\frac{1}{n} \sum_{j=1}^{n} x_{j}^{*} y_{j}^{*}=\frac{1}{n} \sum_{j=1}^{n} \cos \left(\theta+\frac{j 2 \pi}{n}\right) \sin \left(\theta+\frac{j 2 \pi}{n}\right) \\
& =\frac{1}{n} \sum_{j=1}^{n} \frac{1}{2} \sin \left(2\left(\theta+\frac{j 2 \pi}{n}\right)\right)=0,
\end{aligned}
$$

which is identically 0 .

Proposition 1 is helpful in setting up the three ending points in Figure 2. Following Proposition 1 , we choose $n=3, r_{x}=\sigma_{1} \sqrt{2 \Delta t}$, and $r_{y}=\sigma_{2} \sqrt{2 \Delta t}$, where $\sigma_{1}$ and $\sigma_{2}$ are the volatility parameters of the processes $S_{1}(t)$ and $S_{2}(t)$, respectively. These choices ensure the upright ellipse match the volatilities of individual Brownian motions. The next step is to adjust three points along the ellipse to fulfill the correlation between $d W_{1}$ and $d W_{2}$.

To incorporate the correlation between the two Brownian motions, we then rotate the axes, as Figure 3 shows. In Figure 3, the $x$-axis rotates toward $y$ (counterclockwise) and the $y$-axis rotates toward $x$ (clockwise) by $\phi$ degrees. Any three points (say $A^{*}, B^{*}$, and $C^{*}$ ) on the new ellipse, as long as they are "equally spread" according to the new axes, have the identical variances as the unrotated "upright" ellipse and the required correlation. In other words, the rotation of axes by a certain degree uniquely defines a correlation while leaving individual variances unchanged. In an extreme case where $\phi=45^{\circ}$, the two axes collide and the correlation is +1 . In the other extreme, $\phi=-45^{\circ}$ gives the correlation of -1 .

Recall that the problem of mismatching (between unknown states and equations) stems from the fact that the number of correlations grows at a factorial rate while the number of states can grow only at a linear rate. The structure we establish here shows that the correlation between any two assets is uniquely defined by the rotation angle. For $k$ assets, there are always $C_{2}^{k}$ angles (between any two axes) to uniquely match the same number of correlations. Hence, our model no longer suffers from the problem that the number of correlations mismatches the

\footnotetext{
${ }^{7}$ The cancellation of equally spread points is due to the symmetric and oscillating behavior of sine and cosine functions

${ }^{8}$ See footnote 7.
} 
FIGURE 3

Rotation in Two Dimensions

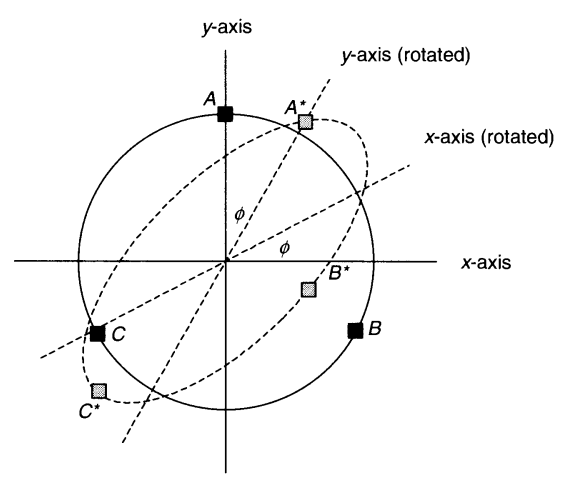

number of assets and it allows us to establish an algorithm for higher dimensional models (i.e., $k>2$ ).

It is important to make sure that the rotation of the axes only incorporates in the correlation and not change the variances of the two Brownian motions. We define the rotation matrix as follows. ${ }^{9}$

Proposition 2. Let $\left(x_{1}, y_{1}\right), \ldots,\left(x_{n}, y_{n}\right)$ be as in Proposition 1 and let $x_{j}^{*}=x_{j} / r_{x}$, $y_{j}^{*}=y_{j} / r_{y}$. Let $\phi$ be an arbitrary constant, interpreted in the sequel as a rotation angle. Define $\left(\hat{x}_{1}, \hat{y}_{1}\right), \ldots,\left(\hat{x}_{n}, \hat{y}_{n}\right)$ by $\left(\hat{x}_{j}, \hat{y}_{j}\right)=\left(x_{j}^{*}, y_{j}^{*}\right) \times \Psi$ where

$$
\begin{aligned}
\Psi & =\left[\begin{array}{ll}
\cos \phi & \sin \phi \\
\sin \phi & \cos \phi
\end{array}\right] \\
& =\frac{1}{\sqrt{1+\tan ^{2} \phi}}\left[\begin{array}{cc}
1 & \tan \phi \\
\tan \phi & 1
\end{array}\right] \quad j=1, \ldots, n
\end{aligned}
$$

Then the corresponding random variables $\hat{X}$ and $\hat{Y}$ satisfy:

$$
\begin{aligned}
E[\hat{X}] & =E[\hat{Y}]=0, \\
\operatorname{var}[\hat{X}] & =\frac{1}{2}, \quad \operatorname{var}[\hat{Y}]=\frac{1}{2}, \\
\rho & =\operatorname{corr}[\hat{X}, \hat{Y}]=\sin (2 \phi) .
\end{aligned}
$$

\footnotetext{
${ }^{9}$ The Cholesky decomposition, which follows the Gaussian elimination principle, is another way of rotating the ellipse. One can verify that the rotated points by the Cholesky method fall on the ellipse. Detailed derivations can be obtained upon request.
} 
Proof.

$$
\begin{aligned}
E[\hat{X}] & =\frac{1}{n} \sum_{j=1}^{n} \hat{x}_{j}=\frac{\cos \phi}{n} \sum_{j=1}^{n} x_{j}^{*}+\frac{\sin \phi}{n} \sum_{j=1}^{n} y_{j}^{*}=0 \\
V[\hat{X}] & =\frac{1}{n} \sum_{j=1}^{n} \hat{x}_{j}^{2} \\
& =\frac{\cos ^{2} \phi}{n} \sum_{j=1}^{n} x_{j}^{* 2}+\frac{\sin ^{2} \phi}{n} \sum_{j=1}^{n} y_{j}^{* 2}+\frac{2 \cos \phi \sin \phi}{n} \sum_{j=1}^{n} x_{j}^{*} y_{j}^{*} \\
& =\frac{1}{2} \cos ^{2} \phi+\frac{1}{2} \sin ^{2} \phi+0=\frac{1}{2} .
\end{aligned}
$$

The expected value and variance of $\hat{Y}$ can be obtained similarly by equation (11),

$$
\begin{aligned}
K[\hat{x}, \hat{y}]= & \frac{1}{n} \sum_{j=1}^{n} \hat{x}_{j} \hat{y}_{j} \\
= & \frac{\cos \phi \sin \phi}{n} \sum_{j=1}^{n} x_{j}^{* 2}+\frac{\cos \phi \sin \phi}{n} \sum_{j=1}^{n} y_{j}^{* 2} \\
& +\frac{2(\cos \phi \cos \phi+\sin \phi \sin \phi)}{n} \sum_{j=1}^{n} x_{j}^{*} y_{j}^{*} \\
= & \frac{1}{2}(\cos \phi \sin \phi+\cos \phi \sin \phi)+0=\frac{1}{2} \sin (2 \phi) .
\end{aligned}
$$

Hence, the correlation can be shown to be only dependent on the rotation angle,

$$
\rho=\frac{K[\hat{X}, \hat{Y}]}{\frac{1}{2}}=\sin (2 \phi)
$$

We now return to the two-dimensional $(k=2)$ stock price process described in the beginning. Using the correlation coefficient $\rho$ of the two Brownian motions, we define $\phi=\left(\sin ^{-1}(\rho)\right) / 2$, and then $\left(x_{1}, y_{1}\right), \ldots,\left(x_{n}, y_{n}\right)$ as in Proposition 2.

Restricting ourselves to $n=3$, we define the discrete time price process as follows. For any given time, $t$, the next period stock prices are

$$
\begin{aligned}
& {\left[\begin{array}{ll}
\ln S_{1,1}(t+\Delta t) & \ln S_{2,1}(t+\Delta t) \\
\ln S_{1,2}(t+\Delta t) & \ln S_{2,2}(t+\Delta t) \\
\ln S_{1,3}(t+\Delta t) & \ln S_{2,3}(t+\Delta t)
\end{array}\right]} \\
& =\left[\begin{array}{l}
1 \\
1 \\
1
\end{array}\right] \times\left[\ln S_{1}(t)+\left(r-\frac{\sigma_{1}^{2}}{2}\right) \Delta t \quad \ln S_{2}(t)+\left(r-\frac{\sigma_{2}^{2}}{2}\right) \Delta t\right] \\
& +\left[\begin{array}{ll}
r_{x} \hat{x}_{1} & r_{y} \hat{y}_{1} \\
r_{x} \hat{x}_{2} & r_{y} \hat{y}_{2} \\
r_{x} \hat{x}_{3} & r_{y} \hat{y}_{3}
\end{array}\right],
\end{aligned}
$$


where $\left(r_{x} \hat{x}_{j}, r_{y} \hat{y}_{j}\right)$ is the coordinate of the three points: for example, $A^{*}, B^{*}, C^{*}$ in Figure 3.

While the extension to higher dimensions $(k>2)$ is easy to visualize, the algebraic details are complex. We provide the necessary results in the Appendix and the complete derivations are available on request.

\section{Numerical Examples}

Although numerical performance is not the main focus of the paper, we find that this model provides as good a performance as other existing lattice models.

The first numerical example is the computation of the value of an exchange option with the closed-form solution introduced by Fischer (1978) and Margrabe (1978). We adopt the following parameter values:

\begin{tabular}{llc}
\hline No. of Steps $(n)$ & Stock $X$ & Stock $Y$ \\
\cline { 1 - 1 } Current price $(X, Y)$ & 40 & 40 \\
Volatility $\left(\sigma_{X}, \sigma_{y}\right)$ & 0.2 & 0.3 \\
Correlation $(\rho)$ & 0.5 & 0.05 \\
Risk-free rate $(r)$ & 7 months \\
Maturity $(T)$ & P & \\
\hline
\end{tabular}

The exchange formula is based upon the maturity payoff of

$$
\max \{X-Y, 0\}
$$

To illustrate our numerical procedure, we first demonstrate our model with a twoperiod example. In this case, $\Delta t=7 \div 12 \div 2=0.29267$. From Proposition 1 and the discussion that follows, we know that the two radiuses of the ellipse for each period can be computed as

$$
\begin{aligned}
& r_{x}=\sigma_{x} \sqrt{2 \Delta t}=0.2 \times \sqrt{2 \times 0.29267}=0.1528, \\
& r_{y}=\sigma_{y} \sqrt{2 \Delta t}=0.3 \times \sqrt{2 \times 0.29267}=0.2291 .
\end{aligned}
$$

The rotation angle is $\left(\sin ^{-1} \rho\right) / 2=\pi / 12$ and hence the rotation matrix is

$$
\frac{1}{\sqrt{1+\tan ^{2} \frac{\pi}{12}}}\left[\begin{array}{cc}
1 & \tan \frac{\pi}{12} \\
\tan \frac{\pi}{12} & 1
\end{array}\right]=\left[\begin{array}{ll}
0.9659 & 0.2588 \\
0.2588 & 0.9659
\end{array}\right] .
$$

Applying this rotation matrix to the coordinates of the points on the circle (values taken from footnote 5), we obtain 


$$
\begin{aligned}
{\left[\begin{array}{l}
A^{*} \\
B^{*} \\
C^{*}
\end{array}\right] } & =\left[\begin{array}{rr}
0 & 1 \\
\frac{\sqrt{3}}{2} & -\frac{1}{2} \\
-\frac{\sqrt{3}}{2} & -\frac{1}{2}
\end{array}\right] \times\left[\begin{array}{rr}
0.9659 & 0.2588 \\
0.2588 & 0.9659
\end{array}\right] \\
& =\left[\begin{array}{rr}
0.2588 & 0.9659 \\
0.7071 & -0.2588 \\
-0.9659 & -0.7071
\end{array}\right] .
\end{aligned}
$$

Then, we apply the radiuses respectively to obtain

$$
\left[\begin{array}{rr}
0.0395 & 0.2213 \\
0.1080 & -0.0593 \\
-0.1475 & -0.1620
\end{array}\right] .
$$

The drift term, which is $\left(r-\left(\sigma^{2} / 2\right)\right) d t$, is equal to 0.00875 and 0.001458 for stocks $X$ and $Y$, respectively. Hence, the three points (log stock price) at time 1 are

$$
\begin{gathered}
{\left[\begin{array}{ll}
3.6889 & 3.6889 \\
3.6889 & 3.6889 \\
3.6889 & 3.6889
\end{array}\right]+\left[\begin{array}{ll}
0.00875 & 0.001458 \\
0.00875 & 0.001458 \\
0.00875 & 0.001458
\end{array}\right]} \\
+\left[\begin{array}{rr}
0.0395 & 0.2213 \\
0.1080 & -0.0593 \\
-0.1475 & -0.1620
\end{array}\right]=\left[\begin{array}{ll}
3.7372 & 3.9117 \\
3.8056 & 3.6310 \\
3.5501 & 3.5283
\end{array}\right]
\end{gathered}
$$

Repeating this computation on all three nodes at time 1 for the second period, we get coordinates for six nodes. Taking exponentials of the values, we obtain three pairs of values for the two stocks at time 1 and six pairs of values for the two stocks at time 2 .

\section{FIGURE 4}

Stock Prices in a Two-Period $(N=2)$, Two-Stock $(k=2)$ Example

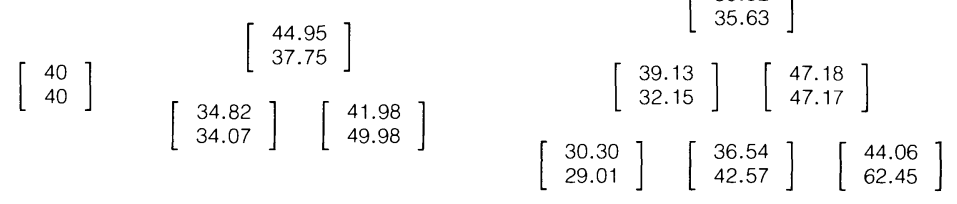


Figure 4 corresponds precisely to Figure 1. For example, point $A$ in Figure 1 is represented by a pair of stock prices $(44.95,37.75)$ in Figure 4 and point $A B$ in Figure 1 is represented by $(47.18,47.17)$ in Figure 4 . The option prices computed using Figure 4 are depicted in Figure 5.

\section{FIGURE 5}

Option Prices in a Two-Period $(N=2)$, Two-Stock $(k=2)$ Example

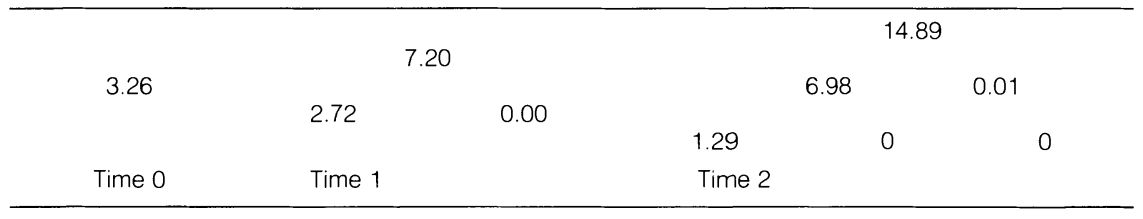

The above option values can be obtained by using equations (1) and (2). Taking the option price at the top state in time 1 as an example, the option value is 7.20 and the stock values in the same state are 44.95 and 37.75 for stocks $X$ and $Y$, respectively. The stock prices in the three resulting states at time 2 are $(47.18$, $47.17),(50.52,35.63)$, and $(39.13,32.15)$. Using equation (1), we can compute the hedge ratios as follows,

$$
\left[\begin{array}{l}
\Delta_{x} \\
\Delta_{y} \\
\Delta_{B}
\end{array}\right]=\left[\begin{array}{lll}
47.18 & 47.17 & 1 \\
50.52 & 35.63 & 1 \\
39.13 & 32.15 & 1
\end{array}\right]^{-1}\left[\begin{array}{l}
0.00 \\
6.98 \\
1.29
\end{array}\right]=\left[\begin{array}{r}
1 \\
-1 \\
0
\end{array}\right],
$$

and then using equation (2), we can compute the option value of 7.20 at the top state at time 1 ,

$$
\left[\begin{array}{lll}
44.95 & 37.75 & 1 \\
1+5 \% \times 0.29267
\end{array}\right] \times\left[\begin{array}{r}
1 \\
-1 \\
0
\end{array}\right]=7.20 .
$$

Repeating this procedure, we obtain two other option values of 2.72 and zero at time 1 and the current option value of 3.26. ${ }^{10}$

As the number of periods gets large, the above tree structure should approximate the bivariate lognormal diffusion process and the option price should converge to the closed-form value. The closed-form solution is

$$
C=X N(d)-Y N(d-v),
$$

\footnotetext{
${ }^{10}$ It should be noted that the same option values can be solved for alternatively by using the usual risk-neutral expectations. For example, the option value at the top state at time 1 can be alternatively solved for by

$$
7.20 \approx \frac{1}{1+5 \% \times 0.29267} \times \frac{0.01+14.89+6.98}{3} .
$$

Note that the answer obtained here is not exactly the same as the one in the text. This discrepancy is due to the approximation errors in the drift terms of equation (4). This discrepancy will vanish as the time interval becomes small. Alternatively, the discrepancy should go away if one adjusts the drift terms so that stocks earn the risk-free return.
} 
where

$$
\begin{aligned}
& d=\frac{\ln X-\ln Y+\frac{v^{2}}{2}}{v}, \\
& v=\sqrt{\left(\sigma_{x}^{2}+\sigma_{y}^{2}-2 \rho \sigma_{x} \sigma_{y}\right) T} .
\end{aligned}
$$

The results are summarized in the following table.

\begin{tabular}{cc}
\hline \multicolumn{1}{c}{$n$} & $\frac{\text { Option Value }}{3.264}$ \\
10 & 3.235 \\
20 & 3.226 \\
50 & 3.219 \\
closed form & 3.219 \\
\hline
\end{tabular}

From the table, we find that the convergence is fast and the accuracy of a 50-step calculation is comparable to the single-factor binomial method.

The second example compares our model with Boyle (1988) who prices the options on the max as follows,

$$
\max \{\max \{X, Y\}-K, 0\} .
$$

All the parameters of the contract are the same as those of the first example. The analytical solution has been solved by Johnson (1987) as a bivariate normal integration. Using the above information about the two stocks and a strike price of 35 , the Boyle model is able to converge in 50 steps to the second decimal place, in a comparison to Johnson's closed-form model. ${ }^{11}$ Our model can converge equally well with fewer nodes. In Boyle, the number of end states in a 50-step lattice is $51^{2}=2601$ but, in our model, the number of end nodes in 50 steps is $51 \times 52 \div$ $2=1326$, about a $50 \%$ improvement. $^{12}$

\begin{tabular}{lcc}
\hline No. of Steps & Boyle Model & Our Model \\
\cline { 2 - 3 } 10 & 9.404 & 9.448 \\
20 & 9.414 & 9.422 \\
50 & 9.419 & 9.419 \\
ciosed form & 9.420 & 9.420 \\
\hline
\end{tabular}

The third example prices European call and put options on the maximum and minimum of three assets. The benchmark is the Boyle, Evnine, and Gibbs

\footnotetext{
${ }^{11}$ See Boyle (1988), Table 3.

${ }^{12}$ For $k$ factors, the number of nodes at the terminal time $N$ is

$$
\sum_{j_{k}=1}^{N+1} \cdots \sum_{j_{1}=1}^{j_{2}} j_{1}=\frac{\Pi_{i=1}^{k}(N+i)}{k !} .
$$

The required memory converges to $1 / k$ ! of that is required in a $k$-dimensional binomial model.
} 
((1989), hereafter BEG) model, which is based on an $n$-dimensional binomial lattice. The analytical solution to the option values is obtained by using Johnson's model (1987). In these examples, we assume $S_{i}=100, X=100, r=10 \%, \sigma_{i}=0.2$, $\rho_{i j}=0.5$, and $T=1$ where $1 \leq i<j \leq 3$. The following table shows the results for European call and put options. Note that as the number of time steps increases, the computed option values of both methods converge toward the analytical solution. Both models converge equally well. However, our model only needs about onesixth end nodes of BEG. For example, in BEG, the number of end states in an 80 -step lattice is $81^{3}=531,441$ but, in our model, the number of nodes in 80 steps is $C_{2}^{83}=81 \times 82 \times 83 \div 6=91,881$.

\begin{tabular}{lcccc}
\hline \multicolumn{1}{c}{ No. of Steps } & \multicolumn{2}{c}{ BEG Model } & & Our Model \\
\cline { 2 - 3 } & \multicolumn{1}{c}{ Maximum } & Minimum & Maximum & \\
European Call & & & 22.643 & Minimum \\
20 & 22.281 & 5.226 & 22.660 & 5.293 \\
40 & 22.479 & 5.237 & 22.664 & 5.263 \\
60 & 22.544 & 5.241 & 22.668 & 5.258 \\
80 & 22.576 & 5.243 & 22.672 & \\
Analytical solution & 22.672 & 5.249 & & 5.259 \\
European Put & & & 0.945 & 7.421 \\
20 & 0.919 & 7.240 & 0.940 & 7.407 \\
40 & 0.925 & 7.323 & 0.937 & 7.410 \\
60 & 0.928 & 7.350 & 0.937 & 7.403 \\
80 & 0.929 & 7.364 & 0.936 & \\
Analytical solution & 0.936 & 7.403 & & \\
\hline
\end{tabular}

Implementation wise, our approach is advantageous especially when solving higher dimension problems because we choose the minimum number of nodes to complete the market. The above numerical examples demonstrate that our model not only preserves the property of complete market and arbitrage-free intuition that has the economic appeal but also provides comparable accuracy and superior computational efficiency to other existing lattice models. ${ }^{13}$

\section{Conclusion}

Previous lattice models that price American style options with multiple risky assets use various optimization procedures to solve the mismatch problem between the number of states and the number of assets. A complete market model, on the other hand, is free from this problem because the number of states always matches the number of assets. We show that the correlation between two assets can be geometrically defined as the rotation angle of the two axes that span the asset space. This allows us to uniquely identify a lattice structure for any number of risky assets without solving an optimization problem as in other numerical studies. In other words, we establish a complete market economy (defined by Duffie and Huang (1985) and He (1989)) that is missing in other numerical models. Hence, we are able to re-create the Cox-Ross-Rubinstein model in a multiasset, complete market economy. The implementation of our model is no more complicated than that of the single factor CRR model.

\footnotetext{
${ }^{13}$ Efficiency can be further improved if we adopt Heston and Zhou's (2000) proposal to smooth out the payoff function prior to maturity. They show that their method works very well with the binomial model.
} 
Finally, we demonstrate with numerical examples that our model presents better memory efficiency. Comparisons with Boyle (1988) and Boyle, Evnine, and Gibbs (1989) show that our model can achieve the same level of accuracy with fewer nodes in the lattice.

\section{Appendix}

\section{A. Proof of Recombination}

We provide a demonstration in a two-dimensional framework while the $k$ dimensional case is available upon request. We show that for any point to which the multiple nodes of the previous time period can move is recombined.

Let the center point in step 3 be $(x, y)$ and the three points in the previous step going toward it be $\left(x_{1}, y_{1}\right),\left(x_{2}, y_{2}\right)$, and $\left(x_{3}, y_{3}\right)$, as shown in Figure A1.

FIGURE A1

Two-Factor Model in Three Steps

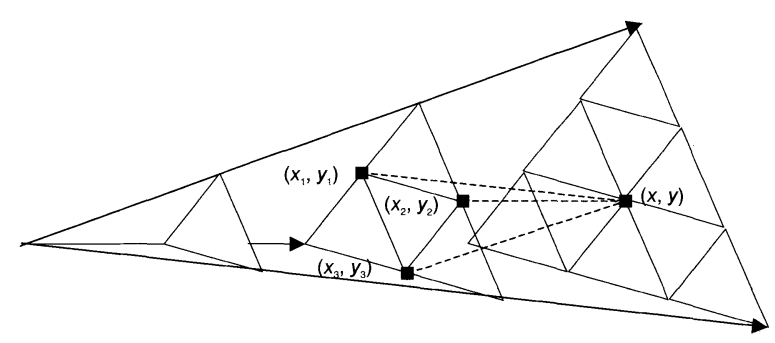

Note that the incremental amounts between the three points in period 2 and the center point in period 3 sum to zero due to the symmetry of Brownian motion. Equal probabilities of the three branches make $(x, y)$ the center of $\left(x_{1}, y_{1}\right),\left(x_{2}, y_{2}\right)$, and $\left(x_{3}, y_{3}\right)$. Hence, by definition, $\left(x_{1}, y_{1}\right),\left(x_{2}, y_{2}\right)$, and $\left(x_{3}, y_{3}\right)$ should recombine at $(x, y)$. Since the log stock price is merely the Brownian motion with drift (which is constant), it is straightforward to show that the values of the each of the stocks at $\left(x_{1}, y_{1}\right),\left(x_{2}, y_{2}\right)$, and $\left(x_{3}, y_{3}\right)$ should also recombine at the node $(x, y)$.

\section{B. Key Results for Implementing Our Model in Higher Dimensions $(k>2)$}

For easy exposition of the high dimensional framework, we first establish a three-factor model. Beyond three factors, we lose visualization but the algorithm we establish in the three-factor model can be well translated. The four states of the three-factor model should form a tetrahedron as follows. 


\section{FIGURE A2}

The Two-Period Geometry of the Three-Factor Model

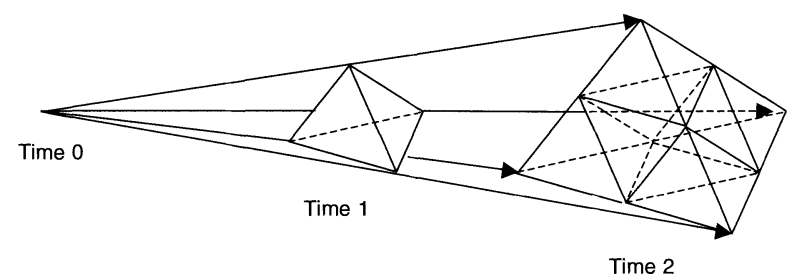

Similar to the two-factor model where the three states are evenly located on the circumference of a circle, the four states in the three-factor model, i.e., the tips of the tetrahedron (or pyramid), must be located evenly on the surface of a "ball." The tetrahedron can spin inside the ball in three-directional space (just like the triangle can spin along the circle).

To demonstrate, we can choose the first point to be on the $z$-axis: $(0,0,1)$. This is a point that is $0^{\circ}$ from the $z$-axis (i.e., $\theta_{z}=0$ ), $90^{\circ}$ from the $x$-axis, and $90^{\circ}$ from the $y$-axis (i.e., $\theta_{x}=\theta_{y}=\pi / 2$ ). With this choice, we still have an infinite number of choices for the remaining three points, since they can travel along the circle that lies $109^{\circ} 28^{\prime}$ from the $z$-axis. ${ }^{14}$ As another convenience, we choose the second point to be along the $y-z$ plane such that the point is $109^{\circ} 28^{\prime}$ from the $z$-axis, $19^{\circ} 28^{\prime}$ from the $y$-axis, and $90^{\circ}$ from the $x$-axis. ${ }^{15}$ The remaining two points are $120^{\circ}$ from the second point in either direction along the circle of $z=-1 / 3 .{ }^{16}$ One can start with a different set of points by turning the hypercube in any direction.

To set up the initial hypercube in high dimensions, we first note that the angle between any two sides of the hypercube is $\cos ^{-1}[-1 / k]$. Hence, a systematic algorithm to choose the $k+1$ points along a $k$-dimensional space can be as follows,

$$
\begin{aligned}
X_{k}^{*}= & {\left[x_{1, j}^{*} x_{2, j}^{*} \cdots x_{k, j}^{*}\right] } \\
= & {\left[\begin{array}{cc}
1 & 0 \ldots 0 \\
\cos \left[\alpha_{k}\right] & \\
\vdots & \sin \left[\alpha_{k}\right] X_{k-1}^{*} \\
\cos \left[\alpha_{k}\right]
\end{array}\right] j=1, \cdots, k+1, }
\end{aligned}
$$

\footnotetext{
${ }^{14}$ The exact degree is $\cos ^{-1}(-1 / 3)$.

${ }^{15}$ Since the angle from the $x$-axis is $90^{\circ}$, the point must be located on the $y-z$ plane. Also since the angle from the $z$-axis is $109^{\circ} 28^{\prime}$, the angle from the $y$-axis must be $109^{\circ} 28^{\prime}-90^{\circ}=19^{\circ} 28^{\prime}$.

${ }^{16}$ Similarly, in high dimensions, as long as the $k+1$ points on the $k$ dimension unit hypercube remain an equal distance from one another, the volatilities remain the same and the correlations are all nil. The angle between any two bonds in the hypercube is $\cos ^{-1}[-1 / k]$. The complete proof of high dimension can be obtained upon request.
} 
where

$$
\begin{aligned}
\alpha_{k} & =\cos ^{-1}\left[-\frac{1}{k}\right], \quad \frac{\pi}{2} \leq \alpha_{k} \leq \pi, \quad \text { and } \\
X_{1}^{*} & =\left[\begin{array}{c}
1 \\
\cos \left[\alpha_{1}\right]
\end{array}\right]
\end{aligned}
$$

One can start with a different set of points by turning the hypercube in any direction. ${ }^{17}$ For higher dimensions $(k>2)$, Propositions 1 and 2 can be extended to the following.

Proposition 1A. The evenly spread $n$ points, where $n \geq k+1$, should yield zero mean, zero covariances, and the variances equal to $(1 / k) r_{i}^{2}$ and $i=1, \ldots, k$.

Proposition $2 A$. The rotation matrix in high dimensions can be written as

$$
\begin{aligned}
& {\left[\hat{x}_{1, j} \hat{x}_{2, j} \cdots \hat{x}_{k, j}\right]=} {\left[x_{1, j}^{*} x_{2, j}^{*} \cdots x_{k, j}^{*}\right] } \\
& \times\left[\begin{array}{cccc}
\frac{1}{\sqrt{A_{1}}} & \frac{\tan \phi_{21}}{\sqrt{A_{1}}} & \cdots & \frac{\tan \phi_{k 1}}{\sqrt{A_{1}}} \\
\frac{\tan \phi_{12}}{\sqrt{A_{2}}} & \frac{1}{\sqrt{A_{2}}} & \cdots & \frac{\tan \phi_{k 2}}{\sqrt{A_{2}}} \\
\vdots & \vdots & \ddots & \vdots \\
\frac{\tan \phi_{1 k}}{\sqrt{A_{k}}} & \frac{\tan \phi_{2 k}}{\sqrt{A_{k}}} & \cdots & \frac{1}{\sqrt{A_{k}}}
\end{array}\right] \\
&=\Psi_{k} X_{k}^{*}, \quad j=1, \cdots, k+1,
\end{aligned}
$$

where

$$
\begin{aligned}
A_{j} & =\sum_{i=1}^{k} \tan ^{2} \phi_{i j}, \\
\phi_{i i} & =45^{\circ},
\end{aligned}
$$

and $\phi_{i j}=\phi_{j i}$ represents the rotation angle between axes $x_{i}^{*}$ and $x_{j}^{*}$.

\footnotetext{
${ }^{17}$ The convergence performance of our method is affected by the position of the hypercube. In other words, it is possible to rotate the hypercube to improve the accuracy of the estimated option prices. Although the best allocation is not known, one can apply the idea of low discrepancy random number generations (see Joy, Boyle, and Tan (1996)) to find a good set of coordinates such that these nodes are distributed evenly.
} 


\section{References}

Amin, K. "On the Computation of Continuous Time Option Prices Using Discrete Approximations." Journal of Financial and Quantitative Analysis, 26 (1991), 477-496.

Barraquand, J., and D. Martineau. "Numerical Valuation of High Dimensional Multivariate American Securities." Journal of Financial and Quantitative Analysis, 30 (1995), 383-404.

$\rightarrow$ Boyle, P. "A Lattice Framework for Option Pricing with Two State Variables." Journal of Financial and Quantitative Analysis, 23 (1988), 1-12.

$\rightarrow$ Boyle, P.; J. Evnine; and S. Gibbs. "Numerical Evaluations of Multivariate Contingent Claims." Review of Financial Studies, 2 (1989), 241-250.

$\rightarrow$ Boyle, P., and Y. Tse. "An Algorithm for Computing Values of Options on the Maximum or Minimum of Several Assets." Journal of Financial and Quantitative Analysis, 25 (1990), 215-227.

$\rightarrow$ Chen, R., and L. Scott. "Pricing Interest Rate Options in a Two Factor Cox-Ingersoll-Ross Model of the Term Structure." Review of Financial Studies, 5 (1992), 613-636.

Clewlow, L., and C. Strickland. Implementing Derivatives Models. New York, NY: John Wiley and Sons (1999).

Cox, J.; S. Ross; and M. Rubinstein. "Option Pricing: A Simplified Approach.” Journal of Financial Economics, 7 (1979), 44-50.

$\rightarrow$ Duffie, D., and C. Huang. "Implementing Arrow-Debreu Equilibria by Continuous Trading of Few Long Lived Securities." Econometrica, 53 (1985), 1337-1356.

$\rightarrow$ Fischer, S. "Call Option Pricing when the Exercise Price Is Uncertain, and the Valuation of Index Bonds." Journal of Finance, 33 (1978), 169-176.

$\rightarrow \mathrm{He}, \mathrm{H}$. "Convergence from Discrete to Continuous Time Contingent Claims Prices." Review of Financial Studies, 3 (1990), 523-546.

Heston, S., and G. Zhou. "On the Rate of Convergence of Discrete Time Contingent Claims." Mathematical Finance, 10 (2000), 53-75.

$\rightarrow$ Ho, T.; R. Stapleton; and M. Subrahmanyam, "Multivariate Binomial Approximations for Asset Prices with Nonstationary Variance and Covariance Characteristics." Review of Financial Studies, 8 (1995), 1125-1152.

$\rightarrow$ Hull, J., and A. White. "Valuing Derivative Securities Using the Explicit Finite Difference Method." Journal of Financial and Quantitative Analysis, 25 (1990), 87-100.

$\rightarrow$ Johnson, H. "Options on the Maximum or the Minimum of Several Assets." Journal of Financial and Quantitative Analysis, 22 (1987), 277-283.

Joy, C.; P. Boyle; and K. S. Tan. "Quasi Monte Carlo Methods in Numerical Finance." Management Science, 42 (1996), 926-938.

Kamrad, B., and P. Ritchken. "Multinomial Approximating Models for Options with $k$ State Variables." Management Science, 37 (1991), 1640-1652.

MaCarthy, L., and N. Webber. "An Icosahedral Lattice Method for Three-Factor Models." Working Paper, Univ. of South Wales and Univ. of Warwick (1999).

$\rightarrow$ Madan, D.; F. Milne; and H. Shefrin. "The Multinomial Option Pricing Model and Its Brownian and Poisson Limits." Review of Financial Studies, 2 (1989), 251-266.

$\rightarrow$ Margrabe, W. "The Value of an Option to Exchange One Asset for Another." Journal of Finance, 33 (1978), 177-186.

Milne, F., and S. Turnbull. “A General Theory of Asset Pricing." Unpubl. Manuscript, Queen's Univ. (1997).

$\rightarrow$ Rendleman, R., and B. Bartter. "Two-State Option Pricing." Journal of Finance, 34 (1979), 10931110.

Sharpe, W. Investments. Englewood Cliffs, NJ: Prentice Hall (1976).

Tavella, D., and C. Randall. Pricing Financial Instruments: The Finite Difference Method. New York, NY: John Wiley and Sons (2000). 